BULL. AUSTRAL. MATH. SOC.

10025,10030

VOL. $25(1982), 313$.

\title{
ON TWO VARIABLE P-ADIC L-FUnCTIONS
}

\author{
RODNEY IAN YAGER
}

Let $E$ be an elliptic curve defined over an imaginary quadratic field $K$ with complex multiplication by the ring of integers of $K$. It has long been felt that certain special values of the complex Hecke $L$-functions attached to powers of the Grossencharacter of the curve $E$ over $K$ are deeply related to the arithmetic of the curve.

Recent results of Katz have shown the existence of two variable $p$-adic $L$-functions which interpolate these special values. The purpose of this thesis is to relate these p-adic L-functions to the arithmetic of the curve $E$. In particular, it will be shown that they are the characteristic power series of certain Iwasawa modules attached to the curve $E$.

Department of Mathematics, Massachusetts Institute of Technology, Cambridge, Massachusetts 02139, USA.

Received 19 January 1982. Thesis submitted to the Australian National University, July 1981. Degree approved January 1982. Supervisor: Professor J. Coates. 